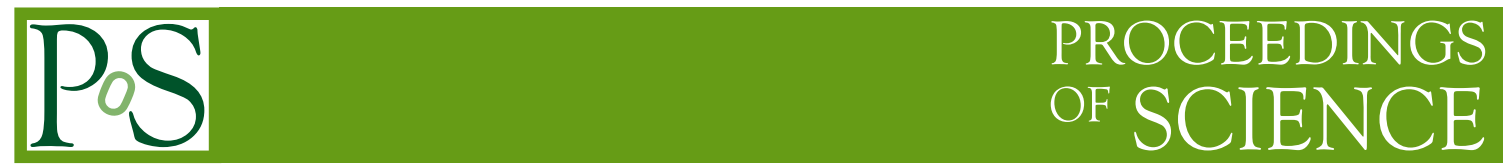

\title{
Future LFV Experiments
}

\section{YOSHITAKA KUNO*i}

Osaka University

E-mail: kuno@phys.sci.osaka-u.ac.jp

Rare muon decay experiments to search for charged lepton flavor violation are described. Future prospects of rare muon decay experiments with highly intense proton accelerators are discussed.

Flavor Physics and CP Violation 2010

May 25-29,2010

Turin, Italy

* Speaker.

${ }^{\dagger}$ A footnote may follow. 


\section{Introduction}

Elementary particle physics has played a key role in expanding our understanding of the fundamental components of matter and the interactions between them. Now, the next important steps toward answering long-standing questions such as "What is the nature of the Universe?", "What is the origin of mass", "How did the Universe begin", and "How did the predominance of matter over antimatters initially appear" are about to be taken in the field of elementary particle physics.

Our understanding of elementary particle physics is based on the Standard Model (SM), which is a gauge theory of the strong and electroweak interactions. The SM has been scrutinized by numerous experimental tests, and it is still consistent with most precision measurements to date. However, the SM cannot answer any of the long-standing questions mentioned above. Therefore, a more complete theory of particle physics has been demanded.

\subsection{Intensity Frontier and Energy Frontier}

There are two experimental approaches that can be used to tackle fundamental questions in elementary particle physics. They are the high-energy frontier approach and the high-precision frontier approach. In the high-energy frontier approach, studies are performed at very high energies, so particle accelerators capable of generating the very highest energies are needed. One example of such an accelerator is the Large Hadron Collider (LHC) at the European Center for Nuclear Research (CERN) in Switzerland.

In the high-precision frontier approach, measurements are made at low energies, but with high precision, and searches for very rare or so-called forbidden processes are performed in order to study phenomena at very high energy scale. Such works are largely based on quantum effects. In the Feynman diagrams entering some rare processes, one occasionally encounters intermediate virtual particles with heavy mass. In some instances, the mass of these particles is so large that they cannot be directly observed in high energy accelerators. It is thus possible in rare processes to study physics phenomena at an energy scale unattainable by using existing particle accelerators.

The physics subject described in this article is about the high precision frontier using muons.

\section{Physics Motivation of cLFV}

In the minimal version of the SM where massless neutrinos are assumed, lepton flavor conservation is a natural consequence of the gauge invariance. Therefore, it has been considered to be a naive explanation on why lepton flavor violation (LFV) in charged leptons in highly suppressed. Charged lepton flavor violation will be referred to "cLFV" from now on.

It have been confirmed that neutrinos are massive and mixed among different neutrino flavor species, by the observation of phenomena of neutrino oscillation. Therefore, lepton flavor for neutrinos is known to be violated. However, cLFV has been yet to be observed, and a discovery of cLFV is considered to be one of the important subjects in elementary particle physics [1].

In the framework of the Standard Model (SM) with massive neutrinos and their mixing, the branching ratio of $\mu \rightarrow e \gamma$ decay $(B(\mu \rightarrow e \gamma))$ can be estimated by

$$
B(\mu \rightarrow e \gamma)=\frac{3 \alpha}{32 \pi}\left|\sum_{l}\left(V_{\mathrm{MNS}}\right)_{\mu l}^{*}\left(V_{\mathrm{MNS}}\right)_{e l} \frac{m_{v_{l}}^{2}}{m_{W}^{2}}\right|^{2},
$$


where $V_{\text {MNS }}$ is the Maki-Nakagawa-Sakata (MNS) matrix elements, $m_{v_{l}}$ and $m_{W}$ are masses of the neutrino $v_{l}$ and the $\mathrm{W}$ boson, respectively. This process is suppressed by the GIM mechanism and the estimated branching ratio is about $10^{-54}$. Therefore, the contribution from the SM with massive neutrinos turns out to be very tiny. As a result, it can be concluded that "(1) observation of cLFV would indicate a clear signal of new physics beyond the SM with massive neutrino.".

The precision measurement of the muon anomalous (g-2) magnetic moment has shown a possible deviation from the SM prediction, up to about 3 sigma. If this is true, it suggests new physics contributions to the dipole interaction of muons with photons. The cLFV processes, such as $\mu \rightarrow e \gamma$ has similar dipole interaction with photons. And it is conceivable that similar new physics contributions would also appear in cLFV. As a result, it can be concluded that "(2) new physics contribution to the muon g-2 would also contributes to cLFV".

Let us consider a general effective Lagrangian for cLFV. It can be given by

$$
\mathscr{L}_{c L F V}=y \frac{e m_{\mu}}{\Lambda^{2}} \bar{\mu}_{R} \sigma^{\mu v} e_{L} F_{\mu v}+\text { h.c. },
$$

where $y$ is a coupling constant of the new interaction, $\Lambda$ is an energy scale of the new interaction, $e$ and $m_{\mu}$ are an electric charge and the muon mass, respectively. $\mu_{R}$ and $e_{L}$ are wave functions for a right-handed muon and a left-handed electron, respectively. $F_{\mu \nu}$ is an electromagnetic field tensor. By using Eq. 2.2), the branching ration of $\mu \rightarrow e \gamma$ at a tree level can be given by

$$
\begin{aligned}
B(\mu \rightarrow e \gamma) & =y^{2} \frac{3(4 \pi)^{3} \alpha}{G_{F}^{2} \Lambda^{4}} \\
& =1 \times 10^{-11} \times\left(\frac{400 \mathrm{TeV}}{\Lambda}\right)^{4}\left(\frac{y}{1}\right)^{2},
\end{aligned}
$$

where $G_{F}$ and $\alpha$ are the Fermi coupling constant and the fine structure constant, respectively. Equation (2.4) indicates that if $y \sim 1$ the search for $\mu^{+} \rightarrow e^{+} \gamma$ at a sensitivity of $10^{-11}$ has a sensitivity to reach an energy scale of $400 \mathrm{TeV}$, which cannot be directly accessed by high energy accelerators. At one loop level, by using the effective Lagrangian in Eq.(2.2), $B(\mu \rightarrow e \gamma)$ can be written by

$$
B(\mu \rightarrow e \gamma)=1 \times 10^{-11} \times\left(\frac{2 \mathrm{TeV}}{\Lambda}\right)^{4}\left(\frac{\theta_{\mu e}}{10^{-2}}\right)^{2},
$$

where $y=g^{2} \theta_{\mu e} /\left(16 \pi^{2}\right)$ and $\theta_{\mu e}$ is a mixing angle between the muon and electron. Equation 2.5 indicates when the energy scale of new physics is $O(1 \mathrm{TeV})$, the mixing angles are suggested to be in an order of $10^{-2}$, which is almost the same magnitudes for those of quarks and neutrinos. Therefore, cLFV is sensitive to new physics at TeV, as the LHC does, and flavor physics in the neutrino and quark sectors. As a result, it can be concluded that "(3) the physics of cLFV is very complementary to that of the LHC.".

In fact, cLFV is known to be sensitive to various extension of new physics beyond the SM. Among them, a well-motivated physics model is a supersymmetric (SUSY) model. FIgure 1 shows one typical diagram of the SUSY contribution to cLFV. In Fig 1 it can be seen that the slepton mixing, which is shown as $\Delta m_{\tilde{\mu} \tilde{e}}^{2}$ (an off-diagonal element of the slepton mass matrix), would introduce cLFV. In the minimum SUSY scenario, the slepton mass matrix is assumed to be diagonal at the Planck scale. At a low energy scale, new physics phenomena such as grand-unification (GUT) or neutrino seesaw would introduce off-diagonal matrix elements such as $\Delta m_{\tilde{\mu} \tilde{e}}$ through quantum 


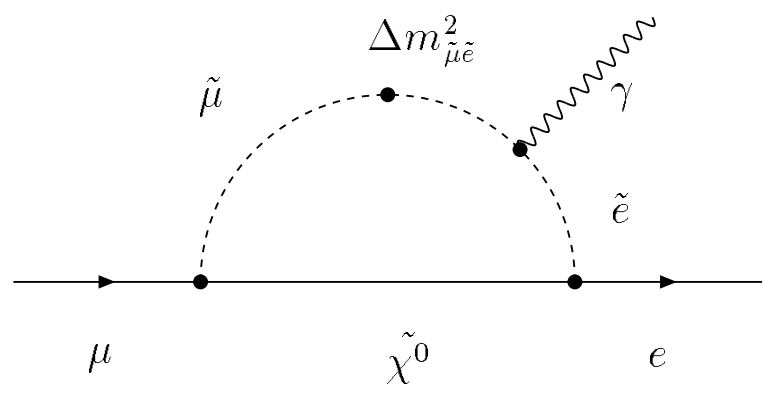

Figure 1: A typical diagram for $\mu \rightarrow e \gamma$.

corrections (renormalization). Therefore, the slepon mixing is sensitive to GUT (at $10^{16} \mathrm{GeV}$ ) or neutrino seesaw mechanism (at $10^{13-14} \mathrm{GeV}$ ). As a result, it can be concluded that "(4) cLFV has potential to study physics at very high energy scale.".

Figure 2 shows predicted branching ratios [2] for muon to electron conversion, which is one of the cLFV processes with muons. In Fig 2 , green and red points indicate the predictions with optimistic and pessimistic parameters respectively, and also the present experimental upper limit is presented. It is seen that if experimental bounds are improved in several orders of magnitude, there is a chance to make discovery of cLFV. As a result, it can be concluded that "(5) theoretical predictions are just below the present experimental bounds".
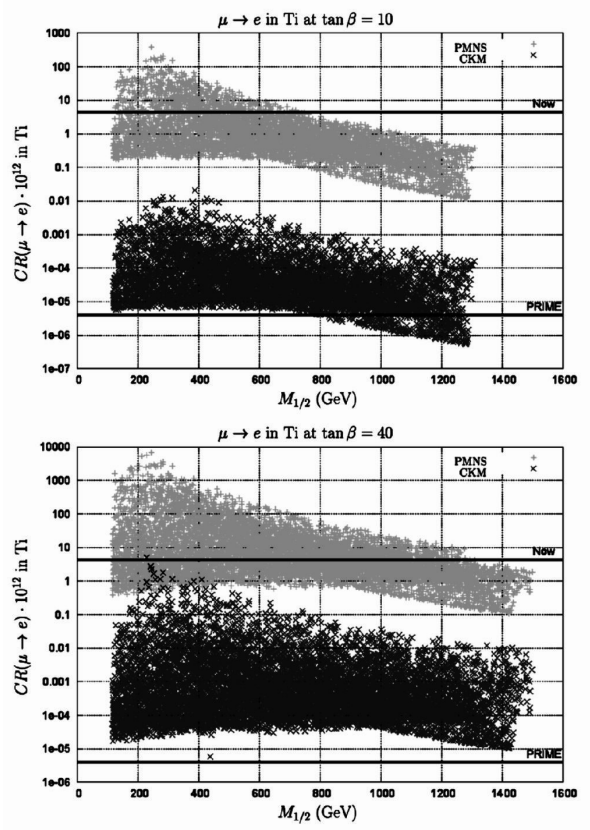

Figure 2: Constained Minimum Supersymmetric Standard Model predictions from Ref.[2].

In a short summary, the five key conclusions, which are mentioned above from (1) to (5), would provide a very strong physics case. 


\section{3. cLFV Experiments}

The experimental upper limits for the cLFV muon processes are summarized in Talbe1 In the following, two typical examples of cLFV processes with muons, namely $\mu^{+} \rightarrow e^{+} \gamma$ and $\mu^{-} N \rightarrow$ $e^{-} N$, are discussed.

\section{1 $\mu^{+} \rightarrow e^{+} \gamma$ Decay}

The event signature of $\mu^{+} \rightarrow e^{+} \gamma$ decay at rest is a positron and a photon are moving backto-back in coincidence with their energies equal to half that of the muon mass $\left(m_{\mu} / 2=52.5 \mathrm{MeV}\right)$. There are two major backgrounds to the search for $\mu^{+} \rightarrow e^{+} \gamma$ decay. One of them is a physics (prompt) background from radiative muon decay, $\mu \rightarrow e v \bar{v} \gamma$, when $e^{+}$and photon are emitted back-to-back with the two neutrinos carrying off a small amount of energy. The other background is an accidental coincidence of an $e^{+}$in a normal muon decay, $\mu \rightarrow e v \bar{v}$, accompanied by a high energy photon. Possible sources of the latter would be either $\mu \rightarrow e v \bar{v} \gamma$ decay, annihilation-inflight or external bremsstrahlung of $e^{+}$s from a normal muon decay.

The present experimental upper limit for $\mu^{+} \rightarrow e^{+} \gamma$ is $1.2 \times 10^{-11}$, which was obtained by the MEGA experiment at Los Alamos National Laboratory (LANL) in the US. A new experiment at PSI called MEG, which aims to achieve a single event sensitivity of $2 \times 10^{-13}$ was built. An improvement factor in a $\mu^{+} \rightarrow e^{+} \gamma$ sensitivity will be expected to utilize a continuous muon beam of $100 \%$ duty factor at PSI. Physics data taking has already started in 2008, obtaining a preliminary result from the 2008 data of $B(\mu \rightarrow e \gamma)<3.0 \times 10^{-11}$.

\section{$3.2 \mu^{-} N \rightarrow e^{-} N$ Conversion in a Muonic Atom}

Another prominent muon cLFV processes is coherent neutrino-less conversion of a negative muon to an electron $\left(\mu^{-} N \rightarrow e^{-} N\right.$ conversion) in a muonic atom. When a negative muon is stopped in some material, it is trapped by an atom, and a muonic atom is formed. After it cascades down energy levels in the muonic atom, the muon is bound in its $1 s$ ground state. The fate of the muon is then either decay in orbit (DIO) $\left(\mu^{-} \rightarrow e^{-} v_{\mu} \bar{v}_{e}\right)$ or nuclear muon capture by a nucleus $N(A, Z)$ of mass number $A$ and atomic number $Z$, namely, $\mu^{-}+N(A, Z) \rightarrow v_{\mu}+N(A, Z-1)$. However, in the context of lepton flavor violation in physics beyond the Standard Model, the exotic process of

Table 1: Experimental limits for the CLFV muon decays.

\begin{tabular}{|l|l|}
\hline Reaction & Present limit \\
\hline$\mu^{+} \rightarrow e^{+} \gamma$ & $<1.2 \times 10^{-11}$ \\
?hline $\mu^{+} \rightarrow e^{*} e^{*} e^{-}$ & $<1.0 \times 10^{-12}$ \\
$\mu^{-} T i \rightarrow e^{-} T i$ & $<4.3 \times 10^{-12}$ \\
$\mu^{-} A u \rightarrow e^{-} A u$ & $<7 \times 10^{-13}$ \\
$\mu^{-} P b \rightarrow e^{-} P b$ & $<4.6 \times 10^{-11}$ \\
$\mu^{+} e^{-} \rightarrow \mu^{-} e^{+}$ & $<8.3 \times 10^{-11}$ \\
\hline
\end{tabular}


neutrino-less muon capture, such as

$$
\mu^{-}+N(A, Z) \rightarrow e^{-}+N(A, Z)
$$

is also expected. This process is called $\mu^{-} N \rightarrow e^{-} N$ conversion in a muonic atom. This process violates the conservation of lepton flavor numbers, $L_{e}$ and $L_{\mu}$, by one unit, but the total lepton number, $L$, is conserved.

The final state of the nucleus $(A, Z)$ could be either the ground state or one of the excited states. In general, the transition to the ground state, which is called coherent capture, is dominant. The rate of the coherent capture over non-coherent capture is enhanced by a factor approximately equal to the number of nucleons in the nucleus, since all of the nucleons participate in the process.

The event signature of coherent $\mu^{-} N \rightarrow e^{-} N$ conversion in a muonic atom is a mono-energetic single electron emitted from the conversion with an energy $\left(E_{\mu e}\right)$ of

$$
E_{\mu e}=m_{\mu}-B_{\mu}-E_{\text {recoil }}
$$

where $m_{\mu}$ is the muon mass, and $B_{\mu}$ is the binding energy of the $1 s$ muonic atom. $E_{\text {recoil }}$ is the nuclear recoil energy which is small and can be ignored. Since $B_{\mu}$ varies for various nuclei, $E_{\mu e}$ could be different. For instance, $E_{\mu e}=104.3 \mathrm{MeV}$ for titanium $(T i)$ and $E_{\mu e}=94.9 \mathrm{MeV}$ for lead $(\mathrm{Pb})$.

From an experimental point of view, $\mu^{-} N \rightarrow e^{-} N$ conversion is a very attractive process in the following reasons.

- The energy of the signal electron of about $105 \mathrm{MeV}$ is far above the end-point energy of the normal muon decay spectrum $(\sim 52.8 \mathrm{MeV})$.

- Since the event signature is a mono-energetic electron, no coincidence measurement is required. The search for this process has the potential to improve sensitivity by using a high muon rate without suffering from accidental background events, which would be serious for other processes, such as $\mu \rightarrow e \gamma$ and $\mu \rightarrow$ eee decays.

There are several potential sources of electron background events in the energy region around 100 $\mathrm{MeV}$, which can be grouped into three categories as follows. The first group is intrinsic physics backgrounds which come from muons stopped in the muon-stopping target. The second is beamrelated backgrounds which are caused by beam particles of muons and other contaminated particles in a muon beam. The third is other backgrounds which are, for instance, cosmic-ray backgrounds, and fake tracking events, and so on.

The previous search for $\mu^{-} N \rightarrow e^{-} N$ conversion was performed by the SINDRUM II collaboration at PSI. The SINDRUM II spectrometer consisted of a set of concentric cylindrical drift chambers inside a superconducting solenoid magnet of 1.2 Tesla. They set an upper limit of $\mu^{-} N \rightarrow e^{-} N$ in $\mathrm{Au}$ of $B\left(\mu^{-}+A u \rightarrow e^{-}+A u\right)<7 \times 10^{-13}$.

\section{Future Experimental Prospects}

Considering its marked importance to physics, it is highly desirable to consider a next-generation experiment to search for cLFV with muons. There are three muon cLFV processes to be considered; namely, $\mu^{+} \rightarrow e^{+} \gamma, \mu \rightarrow e e e$ decays and $\mu^{-} N \rightarrow e^{-} N$ conversion. 
Table 2: Comparison between various cLFV processes with muons.

\begin{tabular}{|l|c|c|c|}
\hline Process & Backgrounds & Beam Requirement & Issue \\
\hline$\mu^{+} \rightarrow e^{+} \gamma$ & accidentals & continuos beam & detector resolutions \\
$\mu \rightarrow e e e$ & accidentals & continuous beam & detector resolutions \\
$\mu^{-} N \rightarrow e^{-} N$ & beam-associated & pulsed beam & beam qualities \\
\hline
\end{tabular}

The three muon LFV processes have different experimental issues that need to be solved to realize improved experimental sensitivities. They are summarized in Table 2 The processes of $\mu^{+} \rightarrow e^{+} \gamma$ and $\mu \rightarrow e e e$ decays are limited by accidental backgrounds. If the incident muon beam rate is increased by a factor $N$, background suppression has to be improved by a factor of $N^{2}$. To achieve this, the detector resolutions have to be significantly improved, which is in general very challenging. In particular, improving the photon energy resolution for $\mu^{+} \rightarrow e^{+} \gamma$ is difficult. On the other hand, for $\mu^{-} N \rightarrow e^{-} N$ conversion, there are no accidental background events, and thus an experiment with higher rates can be performed. If a new muon source with a higher beam intensity and a better beam quality for suppressing beam-associated background events can be constructed, measurements of higher sensitivity can be performed.

Furthermore, it is known that there are more physics processes contributing to $\mu^{-} N \rightarrow e^{-} N$ conversion and a $\mu \rightarrow e e e$ decay than a $\mu^{+} \rightarrow e^{+} \gamma$ decay. Namely, the dipole interaction of photonmediation can contribute to all the three processes, but the box diagrams and four-fermion contact interaction can contribute to only $\mu^{-} N \rightarrow e^{-} N$ conversion and $\mu \rightarrow$ eee decay. In summary, in consideration of the experimental and theoretical aspects, a search for $\mu^{-} N \rightarrow e^{-} N$ conversion would be a natural next choice to accomplish significant improvements in future.

4.1 Next Searches for $\mu^{-} N \rightarrow e^{-} N$ at $10^{-16}$

Next experimental projects to search for $\mu^{-} N \rightarrow e^{-} N$ conversion with a higher sensitivity are being pursued in the USA and Japan. To suppress background events, in particular beamrelated backgrounds, the following key elements have been proposed. They are based on the ideas developed in the MELC proposal at the Moscow Meson Factory. [3]

- Beam pulsing :

Since muonic atoms have lifetimes of the order of $1 \mu \mathrm{sec}$, a pulsed beam with its width that is short compared with these lifetimes would allow one to remove prompt background events by performing measurements in a delayed time window. To eliminate prompt beam-related backgrounds, proton beam extinction is required during the measurement interval.

- High Field Solenoids for Pion Capture :

Superconducting solenoid magnets of a high magnetic field surrounds a proton target to capture pions in a large solid angle. It leads a dramatic increase of muon yields by several orders of magnitude.

- Curved Solenoids for Muon Transport :

The solenoid system for muon transport has high transmission efficiency, resulting a signif- 
icant increase of muon flux. The curved solenoids select charges and momenta of muons as well as removing neutral particles in a beam. The principle is as follows. In a curved solenoidal magnetic field, a center of the helical trajectory of a charged particle is shifted perpendicular to the curved plane. The shift, whose amount is given as a function of momentum and its charge, makes a dispersive beam. By placing appropriate collimators, charges and momenta of muons can be selected.
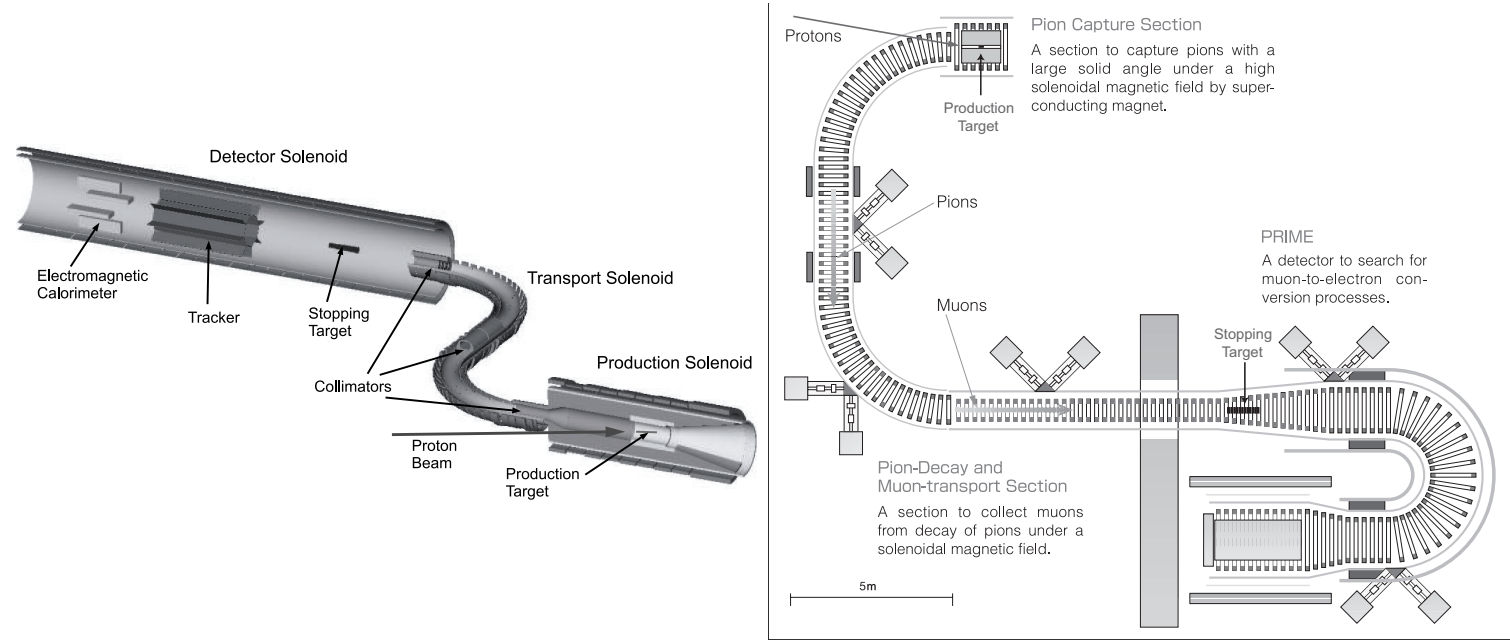

Figure 3: Schematic layout of the Mu2e experiment at FNAL (left) and the COMET experiment at J-PARC (right).

One proposal in the USA was the MECO experiment at BNL. [4] It was mostly based on the MELC design and aimed to search for $\mu^{-} N \rightarrow e^{-} N$ conversion at a sensitivity of better than $10^{-16}$. It consists of the production solenoid system, the transport solenoid system and the detector solenoid system. Unfortunately, the MECO proposal was cancelled in 2005, owing to its funding problems. In 2008 a new initiative at Fermi National Accelerator Laboratory (FNAL), which is called the Mu2e experiment, has been made to perform a MECO-type experiment. [5] The Mu2e experiment is planned to combat beam-related background events with the help of a $8 \mathrm{GeV} / c$ proton beam of $25 \mathrm{~kW}$ in beam power from the Booster machine at FNAL. Figure 4.1 (left) shows the proposed layout of the Mu2e experiment.

The other experimental proposal to search for $\mu^{-} N \rightarrow e^{-} N$ conversion, which is called COMET (COherent Muon to Electron Transition), is being prepared at the Japan Proton Accelerator Research Complex (J-PARC), Tokai, Japan. [6 The COMET uses a proton beam of $56 \mathrm{~kW}$ from the J-PARC main ring. The aimed sensitivity at COMET is similar to Mu2e, better than $10^{-16}$. A schematic layout of the COMET experiment is presented in Figure 4.1(right). The differences of the designs between Mu2e and COMET exist in the adoption of C-shape curved solenoid magnets for both a muon beamline and a $e^{+}$spectrometer in COMET. First of all, in Mu2e, after the first 90-degree bending, the muons of their momenta of interest are necessarily shifted back to the median plane in the second 90-degree bending with opposite bending direction (therefore a S-shape), whereas in COMET, by applying a vertical correction magnetic field, the muons of interest can be kept on the median curved plane. From this fact, any opposite bending direction is not needed 
and a 180-degree bending in COMET would provide larger dispersion to give a better momentum selection. Secondary, a curved solenoid spectrometer in COMET is useful to eliminate low-energy events from muon decays in orbit before going into the detector, resulting in lower single counting rates in the detectors. To eliminate beam-related backgrounds at this sensitivity, the both experiments, Mu2e and COMET, require stringent requirement on proton beam extinction of $10^{-9}$ during the measurement interval. To meet the requirement, additional kicker magnets in the accelerator ring as well as in the extracted proton beam line is being considered.

\subsection{Future Searches for $\mu^{-} N \rightarrow e^{-} N$ at $10^{-19}$}

In the long-term future, significant improvements to aim at an experiment with a $2 \times 10^{-19}$ single-event sensitivity should be considered to make a full coverage of the SUSY parameter space, as seen in Fig 2. The sensitivity of $2 \times 10^{-19}$ can be achieved only together with multi MW proton beam power, which can be provided by future proton accelerators. The other potential key requirements for the improvement are the following.

- Muon beam purity

A low-momentum $(<70 \mathrm{MeV} / \mathrm{c}) \mu^{-}$beam with no pion contamination $\left(<10^{-20}\right)$ would keep prompt background events at a negligible level. This could be achieved by adopting a muon storage ring, where pions decay out during their flight of many turns in the ring. An additional advantage of the method is that heavy muon-stopping targets such as gold, whose muonic-atom lifetimes is around $100 \mathrm{nsec}$, can be studied.

- Narrow energy spread of a muon beam

The $e^{-}$energy resolution is determined by multiple scattering and energy straggling in the muon-stopping target. To improve the resolution. a thiner muon-stopping target is required. To keep a good muon-stopping efficiency, a narrow energy spread of a muon beam is needed.

- Extinction of a muon beam

As discussed, requirements on beam extinction is very stringent. In addition to the proton beam extinction, the extinction of a muon beam in low energy, which might be easier than high-energy protons, would be needed. To achieve this, fast kicker magnets are needed.

In consideration of these requirements, the PRISM (Phase Rotated Intense Slow Muon source) project is being developed in Japan.[7] In the PRISM project, a muon storage ring, which comprises a fixed field alternating gradient (FFAG) ring, is considered. The FFAG ring has large aperture to accept a muon beam of a large size and allows fast acceleration due to a fixed magnetic field. To achieve narrow energy spread, phase rotation, where fast muons are decelerated and slow muons are accelerated by RF fields in the muon storage ring, is adopted. Furthermore, the kicker magnets for injection and extraction to the muon storage ring would serve the muon-beam extinction, although they might be running at a low repetition such as $100-1000 \mathrm{~Hz}$. The time structure of a proton beam should coincide with their repetition. A schematic layout of PRISM and its PRIME detector is shown in Figure 4 


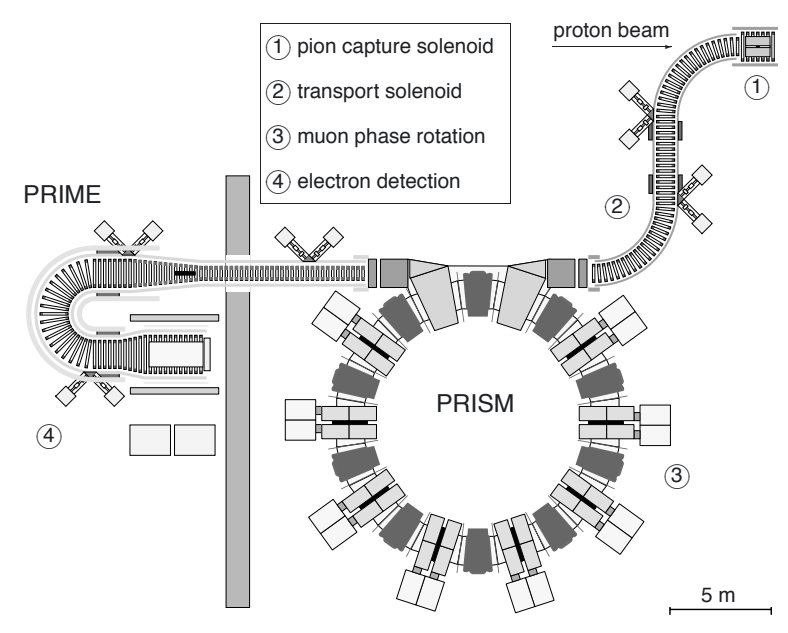

Figure 4: Schematic layout of the PRISM/PRIME detector.

\section{Summary}

The search for CLFV with muons has large potential to obtain hints for new physics beyond the Standard Model. The physics of cLFV has received glowing attention from both theorists and experimentalists. In this article, the phenomenology, the experimental status and future experimental prospects for various cLFV processes with muons are described. Ultimate searches for $\mu^{-} N \rightarrow e^{-} N$ conversion at a single event sensitivity of $2 \times 10^{-19}$ have been considered. Here, high intensity proton accelerator would play a crucial role, since these searches would not be achieved without high intensity proton accelerators of multi MW beam power, such as Project-X. These attempts would offer extraordinary opportunities for exploring new phenomena which would otherwise be directly inaccessible at future high energy colliders.

\section{References}

[1] Y. Kuno and Y. Okada, Rev. Mod. Phys. 73, 151 (2001)

[2] L. Calibbi, A. Faccia, A. Masierro, and S.K. Vempati, Phys. Rev. D74 116002 (2006).

[3] R.M. Dzhilkibaev and V.M. Lobashev, Sov. J. Nucl. Phys. 49384 (1989) [Yad. Fiz. 49622 (1989)]

[4] M. Bachman et al., MECO, BNL Proposal E940 (1997).

[5] R.M. Carry et al. (Mu2e collaboration), "Proposal to Search for $\mu^{-} N \rightarrow e^{-} N$ with a Single Event Sensitivity Below $10^{-16}$, FNAL proposal (2008).

[6] Y. Kuno et al. (COMET collaboration), J-PARC $50 \mathrm{GeV}$ Proton Synchrotron Proposal P21 "A Experimental Search for Lepton Flavor Violating $\mu^{-} N \rightarrow e^{-} N$ Conversion at Sensitivity of $10^{-16}$ with A Slow-Extracted Bunched Proton Beam", unpublished, 2007. see http://j-parc.jp/NuclPart/pac_0801/pdf/Kuno.pdf

[7] Y. Kuno et al. (PRISM collaboration), J-PARC $50 \mathrm{GeV}$ Proton Synchrotron LOI P20 “ An Experimental Search for a $\mu^{-} N \rightarrow e^{-} N$ Conversion at Sensitivity of the Order of $10^{-18}$ with a Highly Intense Muon Source: PRISM", unpublished, 2006. see http://j-parc.jp/NuclPart/pac_0606/pdf/p20-Kuno.pdf 